\title{
THE
}

\section{SOA-FOG: Secure service-oriented edge computing architecture for smart health big data analytics}

\author{
Rabindra K. Barik \\ Harshchandra Dubey \\ University of Rhode Island \\ Kunal Mankodiya \\ University of Rhode Island, kunalm@uri.edu
}

Follow this and additional works at: https://digitalcommons.uri.edu/ele_facpubs

\section{The University of Rhode Island Faculty have made this article openly available.}

Please let us know how Open Access to this research benefits you.

\section{Terms of Use}

This article is made available under the terms and conditions applicable towards Open Access Policy Articles, as set forth in our Terms of Use.

\section{Citation/Publisher Attribution}

Barik, R. B., Dubey, H., \& Mankodiya, K. (2017, November 14-16). SOA-FOG: Secure service-oriented edge computing architecture for smart health big data analytics. 2017 IEEE Global Conference on Signal and Information Processing (GlobalSIP), Montreal, QC, Canada. doi: 10.1109/GlobalSIP.2017.8308688 Available at: http://dx.doi.org/10.1109/GlobalSIP.2017.8308688

This Conference Proceeding is brought to you for free and open access by the Department of Electrical, Computer, and Biomedical Engineering at DigitalCommons@URI. It has been accepted for inclusion in Department of Electrical, Computer, and Biomedical Engineering Faculty Publications by an authorized administrator of DigitalCommons@URI.For more information, please contact digitalcommons-group@uri.edu. 


\title{
SOA-FOG: SECURE SERVICE-ORIENTED EDGE COMPUTING ARCHITECTURE FOR SMART HEALTH BIG DATA ANALYTICS
}

\author{
Rabindra K. Barik ${ }^{1}$,Harishchandra Dubey ${ }^{2}$, Kunal Mankodiya ${ }^{3}$ \\ ${ }^{1}$ KIIT University, Odisha, India (rabindra.mnnit@gmail.com) \\ 2 The University of Texas at Dallas, Richardson, TX-75080, USA (harishchandra.dubey@utdallas.edu) \\ ${ }^{3}$ University of Rhode Island, Kingston, RI-02881, USA (kunalm@uri.edu)
}

\begin{abstract}
The smart health paradigms employ Internet-connected wearables for telemonitoring, diagnosis for providing inexpensive healthcare solutions. Fog computing reduces latency and increases throughput by processing data near the body sensor network. In this paper, we proposed a secure serviceorientated edge computing architecture that is validated on recently released public dataset. Results and discussions support the applicability of proposed architecture for smart health applications. We proposed SoA-Fog i.e. a three-tier secure framework for efficient management of health data using fog devices. It discuss the security aspects in client layer, fog layer and the cloud layer. We design the prototype by using win-win spiral model with use case and sequence diagram. Overlay analysis was performed using proposed framework on malaria vector borne disease positive maps of Maharastra state in India from 2011 to 2014. The mobile clients were taken as test case. We performed comparative analysis between proposed secure fog framework and state-of-the art cloud-based framework.
\end{abstract}

Index Terms- Fog Computing, Service-Oriented Architecture, Smart Health, Big Data Analytics.

\section{INTRODUCTION}

Sharing, storing and processing of public health data requires secure infrastructure. Health data could be analyzed for locating the area with critical issues of diseases so that proper healthcare facilities could be provided. In many cases, propagation of diseases and ailments are somehow related to geographical location, e.g. Zika Virus in Puerto Rico etc.. Fog computing could be leverage for enhanced analysis of real-world data about diseases and other problems along with the locations [1]. Health data are heterogeneous that lead to challenges in integrating it with existing healthcare facilities, interoperability etc. Fog Computing is an emerging solution that provides low-power node for increasing throughput and reducing latency near the edge of various systems at client layer [2]. Fog computing requires less cloud storage and

The research discussed in this manuscript was supported by National Institute of Health Grant: R01MH108641. transmission power for long-term analysis data. Fog computing has been applied successfully in healthcare and smart cities [3] [4] [5]. The present paper has made the following contributions to the secure transmission of health data:

1. Proposed SoA-Fog, a three-tier secure fog computing based framework that allows communication between client layer, fog layers/nodes and cloud layer for enhanced security features for health data sharing in secure and more efficient way.

2. Sketched the prototype development by using win-win spiral model. The interaction between the various services modeled by using Unified Modeling Language (UML) with use case and sequence diagrams.

3. Overlay analysis was performed on malaria vector borne disease positive maps of Maharastra state in India from 2011 to 2014 for mobile clients.

\section{RELATED WORKS}

\subsection{Fog and Cloud Computing}

Cloud computing has provided ample storage and computational infrastructure for data analysis. It facilitated a transition from desktop to cloud servers. Cloud computing along with other web architectures have created an open environment with shared assets [6]. Cloud framework delivered a robust platform in organizations that interrelate tools, technologies and expertise to nurture production, handling and use of geographical data. Many cloud platforms uncover the application functionalities through geospatial web services [7]. This permit clients to query and update different types of cloud services. It also has provisions of a typical tool to assimilate different cloud applications in the software cloud with enterprise SOA infrastructure. Figure 1 (a) shows systems' view of Cloud Framework for sharing and storing of health data [8]. In client-tier layer, there are three types of clients namely mobile, thick and thin. Clients visualize and analyze the geospatial data. Mobile client operates though mobile devices whereas thin clients works on standard web browsers. In thick clients environment, users process or visualize the geospatial data on desktops that requires installation 


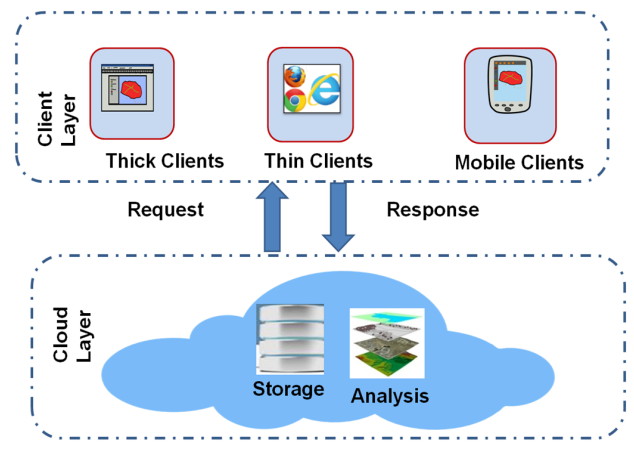

(a)

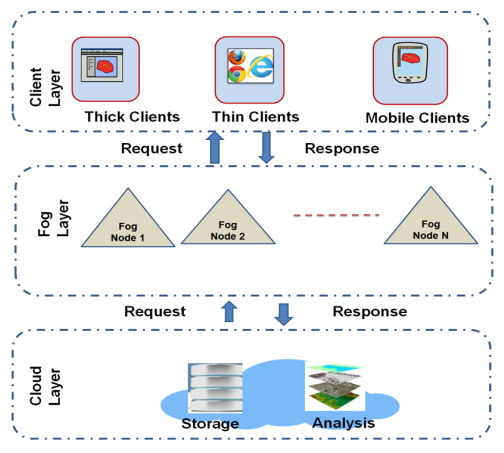

(b)

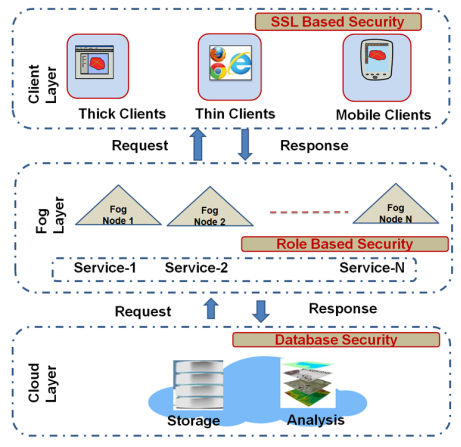

(c)

Fig. 1. (a) Systems' view of Cloud framework with three types of clients (thick, thin and mobile). It has client layer and cloud layer with different web services for analysis and storing of sensitive health data [8]; (b)Conceptual diagram of the Fog framework for power-efficient, low latency and high throughput analysis of the geospatial health data; (c) Conceptual overview of SoA-Fog for enhanced security features

of additional software for full-phase operations [9].The cloud layer is comprised of main geospatial services executed on the servers. It is intermediate between service providers and clients. There are different type of services such as, Web Map Service (WMS), Web Coverage Service (WCS), Web Feature Service (WFS), Web Catalog Service (CSW) and Web Processing Service (WPS) that operates on top of dedicated servers [7]. The detail explanation of every process done by client request, being forward the desire processing service with input of several factors, specifies and provides definite region in leaping box and feedbacks with composite standards. In cloud layer comprises of storing and analysis of the various geospatial data. System utilizes the layer to store, recover, manipulate and update the geospatial data for long-term analysis. With the technology enhancement of fog computing, it has given the more computing power to the cloud framework. Fog framework has three layers as client tier layer, cloud layer and Fog layer. In client tier, the categories of users have been further divided into thick client, thin client and mobile client environment. Processing of geospatial health data can be possible within these three environments. Cloud layer is mainly focused on overall storage and analysis of geospatial data. Fog layer works as middle tier between client layer and cloud layer. It has experimentally validated that the Fog layers are characterized by low power consumption, reduced storage requirement and overlay analysis capabilities.In the Fog layer, all the Fog node developed with Intel Edison processor. Fog framework used to assist and hence enhance the capabilities of cloud framework. In Fog framework, fog node processes the data. After processing, it has the ability to send the data to cloud layer for long-term storage and analysis. So, Fog framework enables the more power to the end-users for better performance without computational overhead at cloud layer. Fog framework added privacy benefit where we process the data locally at Fog devices and send only the analysis results to cloud layer. Figure 1 (b) shows the Conceptual diagram of the fog framework for geospatial health data storage and analysis $[4,10,11]$. From the above conceptual diagram of cloud and fog framework, it observed that the geospatial data as a key components for data analysis in cloud layer $[9,8]$. It requires geospatial data from the various components. It led to the concept of geospatial big data that is discussed in the next section.

\subsection{Geohealth Big Data}

Big data are data those distribution, diversity, scale and timeliness require the use of new technical architectures and analytics to enable insights that unlock new sources of business value. Big data have included data sets with sizes beyond the ability of commonly used software tools to capture, accurate, manage and process data within an acceptable elapsed time $[12,13]$. Big data can come in multiple forms. Most of the big data are semi-structured, quasi structured or unstructured, that requires numerous techniques and tools to analyze and process. Analysis of big datasets can discover the new correlations to spot business trends, combat crime and prevent diseases. Big data sets are growing rapidly because they are increasingly gathered by the information sensing mobile devices, microphones, wireless sensor networks, cameras, aerial images and software logs [14]. As we know that the reliability, manageability and cost saving are the key important factors in that cloud computing always be one of advantageous over other emerge technology for data processing. But in terms of security and privacy are the main concerns for the processing of sensitive data. Particularly in health sector, data are so sensitivity for further processing and analysis [15]. Particularly, for health sector, disease data sharing has been a significant issues for the collaborative preparation, recovery and response stages of numerous disease control mechanism. Disease phenomena are strongly associated with geospatial 
and related temporal factors. For tackling these situation, Cloud framework has provided dynamic and real-time way to represent disease information through the maps on common browsers [2] [4]. So for sharing and analysis of health data in secure way, we have to concentrate with various security issues which has discussed in next section.

\subsection{Security Issues}

With the commencement of cloud computing technology, it has also given so many issues in security and privacy issues. A number of security threats are associated with cloud data services: not only traditional security threats, such as network eavesdropping, illegal invasion, and denial of service attacks, but also specific cloud computing threats, such as side channel attacks, vartualization vulnerabilities, and abuse of cloud services. The following security requirements limit the threats $[16,17]$.

Since fog is deemed as a non-trivial extension of cloud, some security and privacy issues in the context of cloud computing, can be foreseen to unavoidably impact fog computing. Security and privacy issues will lag the promotion of fog computing if not well addressed, according to the fact that 74 percent of IT Executives and Chief Information Officers reject cloud in term of the risks in security and privacy. As fog computing is still in its initial stage, there is little work on security and privacy issues [18]. Since fog computing is proposed in the context of Internet of Things (IoT), and originated from cloud computing, security and privacy issues of cloud are inherited in fog computing [19]. Client authentication, service security and database security are the prime concern in cloud computing environment. By keeping this on mind, it has been proposed a three tier security framework for sharing of health data across the web [20, 21, 22]. From the above related work, it is summarized that, it requires a secure fog computing based framework for sharing and analysis of geospatial big data.

\section{PROTOTYPE DEVELOPMENT}

For the prototype development of SoA-Fog i.e. Fog-based framework, the primary emphasis is on spiral model of Object Oriented Software Engineering (OOSE) method. In OOSE approach of spiral model, the software development process adopts a sequence of steps including requirements prerequisite plan, analysis, development strategy, operation and testing, complete module and framework observation. The process has basically incremental in nature and each implementation refines the analysis and developing stages through evaluation and testing of a completed module. Further, the incremental development strategy of the proposed framework which allows the problem of constructing this framework to be tackled in smaller, more controllable portions of increasing complexity. So there are four phases in that to be defined in SoA-Fog. Phase I deals with the proposed model of SoAFog framework. Phase II describes about the use case and sequence diagram for proposed SoA-Fog. Phase III and Phase IV explain the overlay analysis of the geospatial data on mobile client environment in SoA-Fog framework and comparison analysis for Cloud framework and SoA-Fog framework.

\subsection{Proposed Framework}

This section describes various components of the proposed SoA-Fog framework and discusses the methods implemented in it.The main focus on $S o A-F o g$ has been use of a practical approach to explore and extend the concept of security approaches for Fog computing in health sector. It should provide an effective and efficient means of sharing health related data on the web. Figure 1 (c) shows the proposed three- tier secure service oriented Fog computing framework of health data resources in which the basic over view of service provider, service consumer and catalog service are being shown.

In the SoA-Fog framework, it is proposed to achieve the principle of CIA. Confidentiality can be achieved by SSL based security integration. Role based security is meant to focus on integrity of services whereas database security is to focus on the availability of the data to the authenticated user. In the proposed SoA-Fog framework, it is proposed 3-tier security mechanism, the middle layer i.e. fog layer is technically meant to be role base access control mechanism. The easy to implement mechanisms like discretionary access control mechanism and mandatory access control mechanism can be used. Role base access control mechanism in a preventive way accesses the data tier and ultimately the data will reach the application layer passing through the security mechanism of the data tier and cloud layer. The user role is defined at the very beginning at the client layer by providing authorized access after authenticated verification of the user identity.

In addition, it is expected that each phase would reveal a unique features related to the requirements of infrastructure and enable exploration of the interfaces between fog framework components. The requirements stage of application design aims to specify the behavior of the framework from perspective of a user. From the above defined SoA-Fog framework, it is described the details of use case model and sequence diagram. Figure 2 (b) and 2 (c) have shown the use case and sequence digram of $S o A-F o g$ framework. In the proposed $S o A-F o g$ framework, it is more secure for sharing of health data than cloud based framework. So the next result and discussions section describes about overlay analysis and the comparison analysis of existing cloud framework with SoA-Fog framework by taking suitable parameters.

\section{RESULTS \& DISCUSSIONS}

In this section, data analysis particularly overlay analysis is performed for malaria vector borne disease positive maps of Maharastra, India. It has been found that 2 number of shape files related to malaria information mapping are overlaying 


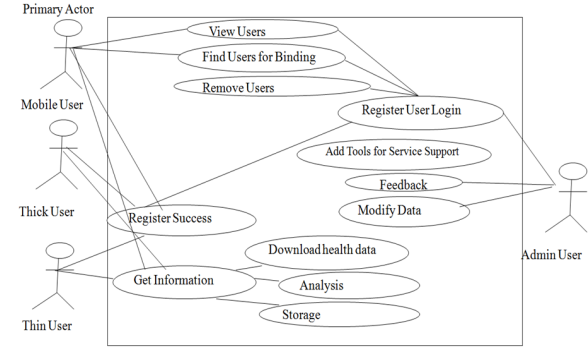

(a)

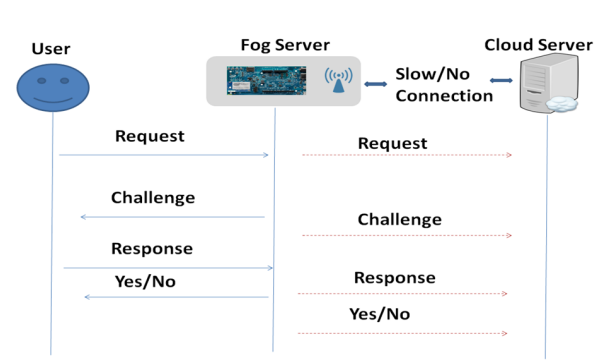

(b)

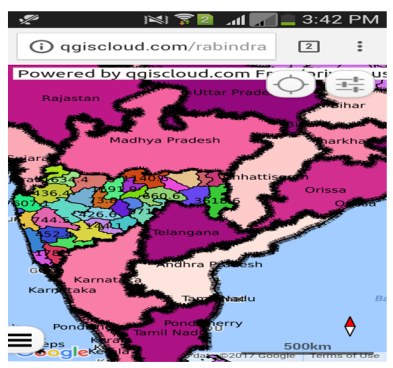

(c)

Fig. 2. (a) Conceptual diagram of the Fog framework for power-efficient, low latency and high throughput analysis of the geospatial health data; (b) Conceptual overview of SoA-Fog for enhanced security features (c) Overlay operation on mobile client environment in qgis cloud [23]

Table 1. Comparison of cloud framework and SoA-Fog Framework

\begin{tabular}{|c|c|c|}
\hline Features & Cloud Framework & SoA-Fog \\
\hline $\begin{array}{l}\text { Bandwidth } \\
\text { Requirements } \\
\text { and Internet } \\
\text { Connectivity }\end{array}$ & $\begin{array}{l}\text { Requires clients to } \\
\text { have network con- } \\
\text { nectivity to the cloud } \\
\text { server and band- } \\
\text { width requirements } \\
\text { grow with the total } \\
\text { amount of health } \\
\text { data generated by } \\
\text { clients. }\end{array}$ & $\begin{array}{l}\text { Operates au- } \\
\text { tonomously to } \\
\text { provide uninter- } \\
\text { rupted services } \\
\text { even no or inter- } \\
\text { mittent Internet } \\
\text { connectivity and } \\
\text { network bandwidth } \\
\text { requirements grow } \\
\text { with total the } \\
\text { amount of data that } \\
\text { need to be process } \\
\text { and sent to the } \\
\text { cloud server after } \\
\text { being authenticated } \\
\text { by the fog layer. }\end{array}$ \\
\hline Size & $\begin{array}{l}\text { Processing has done } \\
\text { with large amount of } \\
\text { health data at a time } \\
\text { and each typically } \\
\text { contains tens of thou- } \\
\text { sands of integrated } \\
\text { servers }\end{array}$ & $\begin{array}{l}\text { Fog node in each lo- } \\
\text { cation can be small } \\
\text { and work with role } \\
\text { based access mech- } \\
\text { anism. }\end{array}$ \\
\hline $\begin{array}{l}\text { Server Loca- } \\
\text { tions }\end{array}$ & $\begin{array}{l}\text { Requires centralized } \\
\text { server in a small } \\
\text { number of big data } \\
\text { centers distributed } \\
\text { environment }\end{array}$ & $\begin{array}{l}\text { Requires } \\
\text { tributed } \\
\text { in many lorvers } \\
\text { tions and over } \\
\text { large geographical } \\
\text { areas, closer to } \\
\text { users along with } \\
\text { fog-to-cloud range }\end{array}$ \\
\hline
\end{tabular}

with google satellite layer. In the present study, it has been used the malaria death mapping data of Maharastra from 2011-2014; has been processing in SoA-Fog.The overlay analysis of various vector data and raster data of particular area has been performed. Initially, the developed datasets have been opened with Quantum GIS; desktop based GIS analysis tools, and performed some join operations in mobile client environment. In Quantum GIS, plugin named as QGISCloud has installed. The said plugin has the capability of storing various raster and vector data set in cloud database for further overlay analysis. After storing in cloud database, it generates the mobile and thin client link for visualization of both vector and raster data set. Figure 2 (c) shows the overlay analysis on mobile client environment. It observes that the overlay analysis is a useful technique for visualization of health data. Both Cloud and SoA-Fog frameworks have specific meaning for a service range with in the cloud computing and client tiers which provide the mutual benefit to each other and interdependent services that leads to the greater storage capacity, control and communication with in the specified range. Table 1 outlines the comparison characteristics of cloud and SoA-Fog framework.

\section{CONCLUSIONS}

In this study, we proposed SoA-Fog framework for enhanced analysis of geo-health data. Intel Edison was used as Fog computer in developed prototypes. Fog devices reduced the storage requirements, transmission power leading to overall efficiency. We performed a case study using Geo-health data of malaria vector borne disease positive maps of Maharastra state in India. We performed the overlay analysis using proposed architecture. In this way, the fog devices add edge intelligence in geo-health data analysis by introducing local processing within cloud-based computing environments.

\section{REFERENCES}

[1] D. Borthakur, H. Dubey, N. Constant, L. Mahler, and K. Mankodiya, "Smart fog: Fog computing framework 
for unsupervised clustering analytics in wearable internet of things," in 5th IEEE Global Conference on Signal and Information Processing, 2017.

[2] Flavio Bonomi, Rodolfo Milito, Jiang Zhu, and Sateesh Addepalli, "Fog computing and its role in the internet of things," in ACM MCC, 2012.

[3] Nicholas Constant etal., "Fog-Assisted wIoT: A Smart Fog Gateway for End-to-End Analytics in Wearable Internet of Things," in IEEE HPCA 2017.

[4] R. K. Barik, H. Dubey, A. B. Samaddar, R. D. Gupta, and P. K. Ray, "Foggis: Fog computing for geospatial big data analytics," arXiv preprint arXiv:1701.02601, 2016.

[5] H. Dubey, N. Constant, A. Monteiro, M. Abtahi, D. Borthakur, L. Mahler, Y. Sun, Q. Yang, and K. Mankodiya, "Fog computing in medical internet-ofthings: Architecture, implementation, and applications," in Handbook of Large-Scale Distributed Computing in Smart Healthcare. 2017, Springer International Publishing AG.

[6] C. Yang, Q. Huang, Z. Li, K. Liu, and F. Hu, "Big data and cloud computing: innovation opportunities and challenges," International Journal of Digital Earth, vol. 10, no. 1, pp. 13-53, 2017.

[7] R. A. AL Kharouf, A. R. Alzoubaidi, and M. Jweihan, "An integrated architectural framework for geoprocessing in cloud environment," Spatial Information Research, pp. 1-9, 2017.

[8] K. Evangelidis, K. Ntouros, S. Makridis, and C. Papatheodorou, "Geospatial services in the cloud," Computers \& Geosciences, vol. 63, pp. 116-122, 2014.

[9] P. Yue, H. Zhou, J. Gong, and L. Hu, "Geoprocessing in cloud computing platforms-a comparative analysis," International Journal of Digital Earth, vol. 6, no. 4, pp. 404-425, 2013.

[10] R. K. Barik, H. Dubey, C. Misra, D. Borthakur, N. Constant, S. A. Sasane, R. K. Lenka, B. S. P. Mishra, H. Das, and K. Mankodiya, "Fog assisted cloud computing in era of big data and internet-of-things: Systems, architectures and applications," in Cloud Computing for Optimization: Foundations, Applications, Challenges, p. 23. Springer, 2018.

[11] R. Barik, H. Dubey, R. K. Lenka, K. Mankodiya, T. Pratik, and S. Sharma, "Mistgis: Optimizing geospatial data analysis using mist computing," in International Conference on Computing Analytics and Networking (ICCAN 2017). Springer, 2017.
[12] J. Andreu-Perez, C. C. Y. Poon, R. D. Merrifield, S. T. C. Wong, and G. Yang, "Big data for health," IEEE journal of biomedical and health informatics, vol. 19, no. 4, pp. 1193-1208, 2015.

[13] R. K. Lenka, R. K. Barik, N. Gupta, S. M. Ali, A. Rath, and H. Dubey, "Comparative analysis of spatialhadoop and geospark for geospatial big data analytics," in 2 nd International Conference on Contemporary Computing and Informatics (IC3I 2016). IEEE, 2016.

[14] J. Lee and M. Kang, "Geospatial big data: challenges and opportunities," Big Data Research, vol. 2, no. 2, pp. 74-81, 2015.

[15] H. Dubey, J. Yang, N. Constant, A. M. Amiri, Q. Yang, and K. Makodiya, "Fog data: Enhancing telehealth big data through fog computing," in Fifth ASE BigData 2015, Kaohsiung, Taiwan. ACM.

[16] J. Tang, Y. Cui, Q. Li, K. Ren, J. Liu, and R. Buyya, "Ensuring security and privacy preservation for cloud data services," ACM Computing Surveys (CSUR), vol. 49, no. 1, pp. 13, 2016.

[17] M. Hafner and R. Breu, "Basic concepts of soa security," Security Engineering for Service-Oriented Architectures, pp. 27-45, 2009.

[18] R. Deng, R. Lu, C. Lai, T. H. Luan, and H. Liang, “Optimal workload allocation in fog-cloud computing towards balanced delay and power consumption," IEEE Internet of Things Journal, 2016.

[19] C. Perera, Y. Qin, J. C. Estrella, S. Reiff-Marganiec, and A. V. Vasilakos, "Fog computing for sustainable smart cities: A survey," arXiv preprint arXiv:1703.07079, 2017.

[20] S. Yi, Z. Qin, and Q. Li, "Security and privacy issues of fog computing: A survey," in International Conference on Wireless Algorithms, Systems, and Applications. Springer, 2015, pp. 685-695.

[21] Z. Yu, M. H. Au, Q. Xu, R. Yang, and J. Han, “Towards leakage-resilient fine-grained access control in fog computing," Future Generation Computer Systems, 2017.

[22] R. K. Barik, H. Dubey, R. K. Lenka, N.V.R. Simha, S. A. Sasane, C. Misra, and K. Mankodiya, "Fog computingbased enhanced geohealth big data analysis," in 2017 International Conference on Intelligent Computing and Control (I2C2). IEEE, 2017.

[23] "Available on : http://qgiscloud.com/rabindrabarik2016/ malaria?mobile=true," Accessed on: 13th February 2017. 